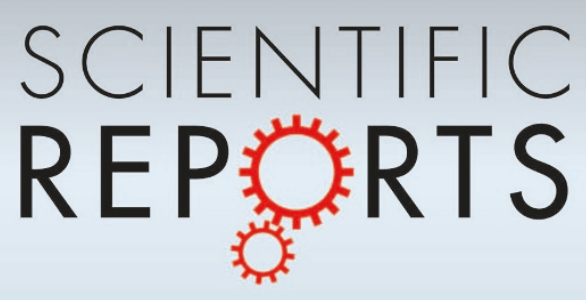

OPEN

SUBJECT AREAS:

CANCER IMAGING

IMAGING STUDIES

KINASES

NEAR-INFRARED SPECTROSCOPY

Received

28 December 2012

Accepted

5 April 2013

Published

22 April 2013

Correspondence and requests for materials should be addressed to

S.A. (achilefus@mir. wustl.edu)

\footnotetext{
* These authors contributed equally to this work.
}

\title{
Dual fluorescent molecular substrates
} selectively report the activation, sustainability and reversibility of cellular PKB/Akt activity

\author{
Duanwen Shen ${ }^{*}$, Mingfeng Bai ${ }^{1 *}$, Rui Tang ${ }^{1 *}$, Baogang $\mathrm{Xu}^{1}$, Xiaoming Ju' ${ }^{4}$, Richard G. Pestell ${ }^{4,5}$ \\ \& Samuel Achilefu' ${ }^{1,2,3}$
}

\begin{abstract}
'Departments of Radiology, ${ }^{2}$ Departments of Biomedical Engineering, ${ }^{3}$ Departments of Biochemistry \& Molecular Biophysics, Washington University in St. Louis, Missouri 631 10, USA, ${ }^{4}$ Departments of Cancer Biology, ${ }^{5}$ Departments of Medical Oncology, Kimmel Cancer Center, Thomas Jefferson University, 233 South 10th Street, Philadelphia, PA 19107.
\end{abstract}

Using a newly developed near-infrared (NIR) dye that fluoresces at two different wavelengths (dichromic fluorescence, DCF), we discovered a new fluorescent substrate for Akt, also known as protein kinase B, and a method to quantitatively report this enzyme's activity in real time. Upon insulin activation of cellular Akt, the enzyme multi-phosphorylated a single serine residue of a diserine DCF substrate in a time-dependent manner, culminating in monophospho- to triphospho-serine products. The NIR DCF probe was highly selective for the Akt1 isoform, which was demonstrated using Akt1 knockout cells derived from MMTV-ErbB2 transgenic mice. The DCF mechanism provides unparalleled potential to assess the stimulation, sustainability, and reversibility of Akt activation longitudinally. Importantly, NIR fluorescence provides a pathway to translate findings from cells to living organisms, a condition that could eventually facilitate the use of these probes in humans.

rotein kinases regulate diverse cellular processes, including biochemical reactions and molecular interactions. The overexpression of kinases is also associated with a host of diseases, such as cancer, cardiovascular diseases, diabetes, and inflammation ${ }^{1}$, raising interest in the use of their signaling pathways as important drug targets ${ }^{2,3}$. Protein kinase B, also known as Akt, has a particularly interesting signaling pathway because it is the central hub for some pathophysiological processes ${ }^{4}$. Recent studies have shown that Akt exists in three isoforms with high structural homology: Akt1, Akt2, and Akt3. However, each isoform has been shown to play distinct, and sometimes contradictory, roles in normal biological and pathological events 5 .

These interesting findings have driven the establishment of in vitro assays to determine the functional status and expression levels of Akt isoforms in cells and tissues. Although many endogenous Akt substrates are known, most share tertiary structure and amino acid sequence homology (i.e., they are non-isoform specific) ${ }^{6}$. Therefore, researchers rely on isoform-specific antibodies to decipher the roles of each kinase subtype. Determining Akt activity is traditionally accomplished by western blotting and ELISA, which are used to detect phosphorylation at Thr308 and Ser473. Unfortunately, these techniques require multiple steps to determine whether a single phosphorylation event has occurred ${ }^{7}$.

In addition to the challenges of generating reproducible antibodies with low cross-reactivity, the cell lysis or fixed cell approach used with these antibodies lacks the ability to capture the dynamics and multidirectional cell signaling pathways that occur in cellular environments ${ }^{7}$. In principle, cell assays could overcome the above limitations, but the widely used methods typically require the encoding of FRET-based fluorescent or bioluminescent proteins that may confine their use to preclinical studies. Moreover, the FRET technique has several problems: the need for optimal distance (typically 1-10 nm) and spectral overlap between donor and acceptor chromophores, the use of two or more fluorescent proteins that could alter the inherent biological properties of the enzyme, and the existence of autofluorescence caused by excitation of proteins in the visible wavelengths $s^{8,9}$. Although fluorescent organic dyes are widely used for biological imaging, the current assays with these molecular probes do not combine the diverse desirable characteristics of a protein kinase assay. In addition, the development of Akt isoform-specific small molecular probes is challenging. These challenges attest to the need for a versatile, 
Akt-specific molecular probe with high sensitivity, high-throughput, and real-time reporting capabilities in live cells and potentially in living organisms.

Recently, we discovered that certain near-infrared (NIR) dyes derived from cypate emit fluorescence at two different wavelengths (approximately $700 \mathrm{~nm}$ and $800 \mathrm{~nm}$ ) along with two corresponding distinct fluorescence lifetimes ${ }^{10}$. Although the mechanism of the dual (dichromic) fluorescence is not fully understood at this time, we found that the induction of structural asymmetry in some carbocyanine molecules amplifies the dichromic fluorescence (DCF) phenomenon. On this basis, we postulated that structural transformation of the NIR cyanine dyes from symmetrical to less symmetrical molecules, or vice versa, could alter the relative intensities of the DCF, thereby providing a unique contrast mechanism to quantitatively detect, image, or monitor the activities of biomolecules in cells and tissues. In this study, the biological relevance of this concept was demonstrated by using Akt to induce and modulate the DCF. This study also identified a molecular probe that is highly specific for the Akt1 isoform.

\section{Results}

Phosphorylation of certain NIR fluorescent dyes induces dual NIR fluorescence. Kinases, such as serine/threonine kinases, are ideal for exploring the DCF mechanism because phosphorylation of their substrates can induce significant structural asymmetry that favors DCF. Therefore, we first prepared a series of compounds that exhibit DCF based on the structural template of a bifunctional NIR fluorescent dye molecule, cypate ${ }^{11}$, and serine, which possesses a hydroxyl group for phosphorylation (Figure 1a). The reaction of serine with cypate resulted in the compound cypate-diserine (LS456). We then prepared a control cypate derivative, LS542, which has the same structural features as LS456, but each of the hydroxyl groups of the serine residues was blocked with tert-butyl to prevent subsequent phosphorylation. We also prepared and evaluated the DCF properties of cypate-monophosphoserine (LS455) and cypate-diphosphoserine (LS631), which mimic the mono- and di-phosphorylation products of LS456 (Figure 1a). All of the products were purified with a semi-preparative HPLC system and characterized by analytical HPLC, a high-resolution mass spectrometer, and spectroscopic methods (see Supplementary Methods).

Spectral analysis of LS542, LS455, LS456, and LS631 were conducted in methanol (Figure 1b). Their optical properties, including the quantum yield, absorption coefficient, and photostability, are summarized in Table 1 and Supplementary Figure 1. LS542, LS456, and LS631, which have more structural symmetry than LS455, exhibit major and smaller emission peaks at approximately $810 \mathrm{~nm}$ and $710 \mathrm{~nm}$, respectively [These peaks are denoted as $700 \mathrm{~nm}$ (or green) and $800 \mathrm{~nm}$ (or red) emissions throughout this paper]. Conversely, the nonsymmetrical LS455 shows a weak emission at $800 \mathrm{~nm}$, but strong fluorescence at $700 \mathrm{~nm}$, which is a reversal of the observed spectral profile for the compounds. A variety of factors may contribute to this observation, including the formation of aggregates, reduction in iminium charge delocalization along the polymethine chain, dimerization, or the existence of two interconvertible molecules at equilibrium in the ground state. The exact mechanism of the observed DCF is under further investigation. However, empirical evidence strongly suggests that the transformation of LS456 to LS455 significantly enhances the $700 \mathrm{~nm}$ emission, which will be useful for reporting the functional status of the target enzyme. A first step toward accomplishing this transformation in a biological setting is to identify a kinase that can utilize LS456 as a substrate.

Akt phosphorylates LS456 in vitro. LS456 was used as a model compound because its two serine residues are highly amenable to phosphorylation. We first investigated the stability of the $700 \mathrm{~nm}$ and $800 \mathrm{~nm}$ emissions of LS456 and its synthetic phosphorylation product, LS455, in tris saline buffer at $30^{\circ} \mathrm{C}$ in buffers for $240 \mathrm{~min}$ under different conditions. Although some fluctuations were noted in the spectral profile of LS455 at the early time points, the ratios of the 700/800 nm emissions for LS455 and 456 were stable over time (Supplementary Figure 1), indicating that subsequent fluorescence ratiometric changes at these wavelengths could be attributed to a target biological event.

We next assessed the selective phosphorylation of the putative fluorescent substrate, LS456, by a subfamily of serine/threonine kinases: PKA, Akt, and PKC in the presence of ATP (a phosphate source). Phosphorylation of LS456 was monitored in the 700-nm channel, where fluorescence intensity enhancement was expected. We found that treatment of LS456 with Akt resulted in a significant fluorescence increase at $700 \mathrm{~nm}$ (Figure 2a), whereas the rate of fluorescence change remained practically unaltered for PKA and even decreased for PKC (Supplementary Figure 2). This result suggests that LS456 was transformed by Akt. However, we found that red fluorescence generally decreased for all the enzymes, albeit in a kinase-specific manner. Because LS456 exhibited the anticipated spectral response to phosphorylation, we further assessed its behavior in the presence of the other serine/threonine kinases, p70S6K and RSK1, as well as a tyrosine protein kinase (PI3K), to establish specificity of the observed 700-nm fluorescence enhancement under similar conditions as Akt. As with the PKA and PKC results, there was no significant increase in the 700-nm emission with any of these other kinases. A significant decrease was even observed for p70S6K at the $700 \mathrm{~nm}$ emission, with a small increase in the 800-nm channel (Supplementary Figure 3). Deciphering the consequence of this unique spectral shift in the presence of p70S6K is not the focus of the current study. Nevertheless, when taken together, the above in vitro results demonstrate that Akt enhances the DCF change of LS456 by increasing the 700-nm emission, which strongly suggests the occurrence of structural transformation of the molecular probe through phosphorylation. The specificity of the LS456-Akt molecular recognition was further confirmed by the lack of a 700-nm fluorescence increase when other serine/threonine kinases (PKA, PKC, p70S6K and RSK1) or tyrosine kinase (PI3K) were used under similar conditions as in the Akt assays.

Replacement of LS456 with the negative control compound, LS542, in the above assays resulted in a significant fluorescence decrease in both the 700-nm and 800-nm channels in the presence of PKA, Akt, and PKC (Figure 2a and Supplementary Figure 2 bottom panel). The hydroxyl group of serine in LS542 is blocked with a tert-butyl group to prevent phosphorylation. Therefore, the correlated trend in fluorescence decrease in both channels could be attributed to the spontaneous aggregation of the highly hydrophobic LS542 in aqueous medium, which is different from the DCF effect observed with LS456.

Cell lysates demonstrate Akt phosphorylation of LS456. After demonstrating the preferential phosphorylation of LS456 by Akt relative to other kinases, we used the DCF approach to monitor Akt activity in cell lysates. Previous studies have shown that the binding of insulin to its cell surface receptor stimulates phosphoinositide-3 kinase (PI3K), which then produces the lipid second messenger phosphatidylinositol-3,4,5-trisphosphate (PIP3) ${ }^{2}$. PIP3, in turn, activates Akt and downstream effectors ${ }^{12,13}$. The breast cancer cell line MCF-7 is widely used to assess Akt activation by insulin. In this study, MCF-7 cells were first transfected with either LS456 or LS542 using GeneJuice transfection reagent and were then incubated with $150 \mathrm{nM}$ insulin to activate $\mathrm{Akt}^{14}$. The cells were homogenized, and the supernatant was placed in a tube and imaged in both the 700$\mathrm{nm}(\mathrm{Ex} / \mathrm{Em} 633 \mathrm{~nm} / 670-730 \mathrm{~nm}$, green color) and 800-nm (Ex/Em $785 \mathrm{~nm} / 805-830 \mathrm{~nm}$, red color) channels. We used two different 

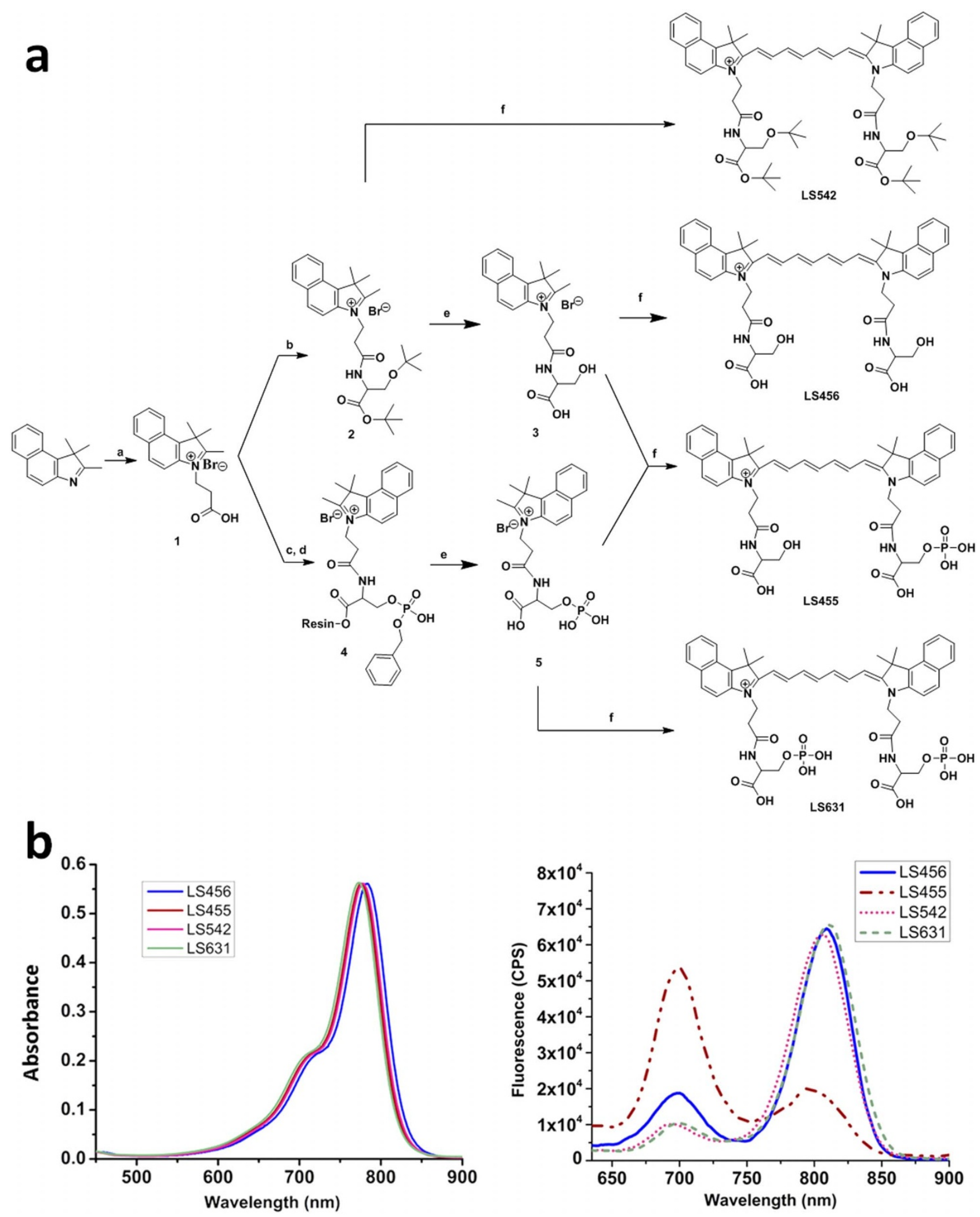

Figure 1 Structures and spectra of molecular reporter probe and controls. (A) Synthesis of LS456, LS455, LS542, and LS631. Reagents and conditions: (a) 3-Bromopropionic acid in dichlorobenzene, $120^{\circ} \mathrm{C}$; (b) DIC ( $N, N$-diisopropylcarbodiimide) and HOBT (hydroxybenzotriazole) in DMF (dimethylformamide) and $\mathrm{H}-\mathrm{Ser}(\mathrm{tBu})-\mathrm{OtBu} \mathrm{HCl}$; (c) Fmoc-Ser $[\mathrm{PO}(\mathrm{OBzl}) \mathrm{OH}] \mathrm{OH}$ was loaded onto Br-Wang resin with cesium iodide and DIEA ( $N$, $N$ diisopropylethylamine); (d) DIC and HOBT in DMF; (e) 95\% TFA/5\% $\mathrm{H}_{2} \mathrm{O}$; (f) $\mathrm{N}$-(5-anilino-2,4-pentadienylidene)aniline hydrochloride, acetic anhydride, DCM, MeOH, and sodium acetate (see the Methods section for details). LS455 is a model of LS456 after phosphorylation. The control LS542 probe has the hydroxyl phosphorylation site of serine blocked with tert-butyl group. (B) Absorption (left) and emission (right) spectra of LS456, LS455, LS542 and LS631 in methanol. Absorbance units were kept below 0.2 for fluorescence measurements. The fluorescence spectrum of LS455 demonstrates the expected spectral shift when a kinase phosphorylates LS456. CPS, photon counts per second. 
Table 1 | Spectral properties of LS455, LS456, LS542, and LS631 in methanol

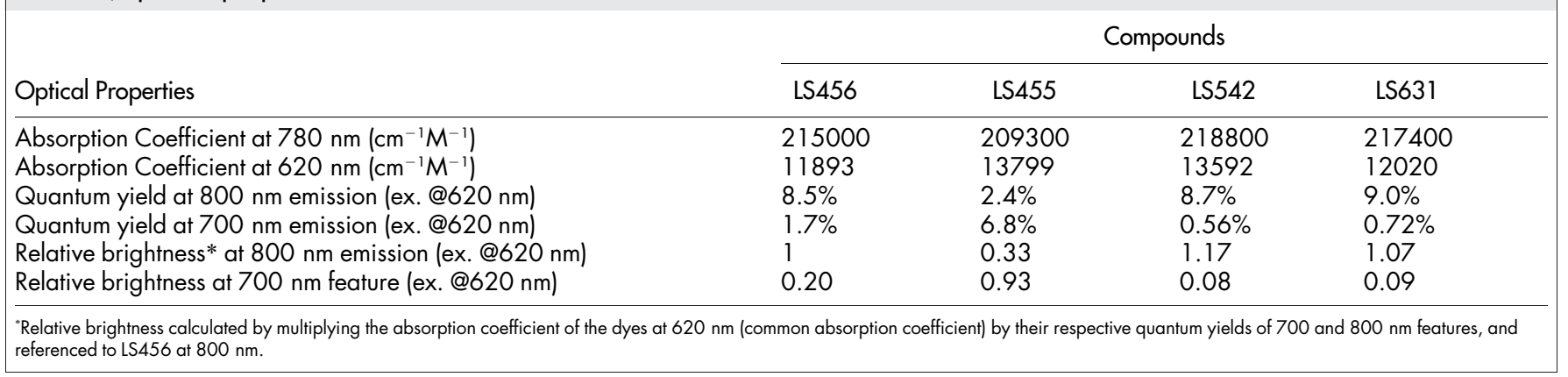

lasers to excite the probes sequentially because our confocal microscope is equipped with a limited number of NIR lasers in the NIR window $(633 / 785 \mathrm{~nm})$. Although we were able to simultaneously obtain dual emissions with $620 \mathrm{~nm}$ excitation in solution-based spectroscopy studies, the brightness for the $800 \mathrm{~nm}$ emission in cells was relatively weak at $633 \mathrm{~nm}$ excitation. After excitation at $633 \mathrm{~nm}$ and $785 \mathrm{~nm}$, the fluorescence image of cell lysates treated with LS456 clearly showed the transformation of the normally red to green fluorescence, which is similar to the spectral properties of the monophosphoserine compound, LS455 (Figure $\mathbf{1 b}$ and 2b). In contrast, with the negative control compound LS542, the red emission was exclusively observed, indicating the absence of a structural transformation of the compound.

To correlate the DCF spectral change with Akt phosphorylation, we used a co-immunoprecipitation (Co-IP) method with a monoclonal anti-phosphorylated $\mathrm{Akt}^{473}$ antibody to isolate the Akt protein-substrate complex, followed by double MS treatment of the residue (MS/MS analysis). We found a time-dependent phosphorylation of LS456. The monophosphorylated derivative of LS456 $(\mathrm{M} / \mathrm{M}+\mathrm{H} 879.2 \mathrm{Da})$ was the major product after $20 \mathrm{~min}$ of insulin activation, which is the same as LS455. Although previous reports demonstrated that Akt typically phosphorylates one serine in the presence of two or more serine residues ${ }^{15,16}$, after $20 \mathrm{~min}$, we also observed significant molecular ions at 957.60/959.60 Da corresponding to the two phosphate groups of LS456. At $45 \mathrm{~min}$ of insulin activation, the peaks for the mono- and di-phosphoserine products disappeared in favor of a triphosphoserine adduct of LS456 (Supplementary Figure $4 \mathrm{~b}$ ). The significant decrease in the abundance of the diphosphoserine molecular ion peak and subsequent dominance of the triphosphoserine ion after $45 \mathrm{~min}$ of insulin activation suggests that this product was derived from the diphosphoserine derivative. If phosphorylation occurred at each of the serine residues of LS456, the diphospho-diserine should have a similar structure as LS631 (Figure 1a). Based on the DCF mechanism, such symmetrical structure would regenerate a red signal as the molecules convert from the green monophosphoserine LS455 to LS631. Instead, the rate of the green fluorescence change increased over time, while that of the red emission decreased correspondingly (Supplementary Figure 2b).

The enhancement of the 700-nm fluorescence most likely suggests that the second phosphorylation occurred at the monophosphoserine residue to form a pyrophosphoserine derivative. Similarly, the combined reduction in the pyrophosphate molecular ion abundance and the further enhancement of the green fluorescence at time points of long insulin activation strongly suggests that the predominant triphosphate group was added to the same serine residue. Otherwise, phosphorylation of the second serine would decrease the 700 -nm emission while increasing the 800 -nm emission, which was not observed. This is the first evidence of the ability of Akt kinase to triphosphorylate its substrate.

To further explore the optical properties of the phosphoserine derivatives, we evaluated LS455 and LS631 in the presence of the serine/threonine kinases PKA, Akt, and PKC under similar conditions as described for LS456 above. While all of the assays showed fairly constant or a significant decrease in the fluorescence signal for both compounds with all the enzymes, only Akt generated a 3-fold fluorescence increase of the 700-nm emission, with a concomitant decrease in the $800-\mathrm{nm}$ channel when LS455 was used (Supplementary Figure 5). Because there was a large DCF change for LS455 in MCF-7 cells under similar conditions as LS456, the lysate was further evaluated by MS/MS and spectroscopic analyses after co-IP. In addition to the starting LS455 and the hydrolysis product (LS456), the data show that additional phosphorylation of LS455 occurred. In particular, spectroscopic and MS/MS analysis of the isolated products bound to $\mathrm{Akt}^{473}$ showed that one and two phosphate groups were present (Supplementary Figure 5 top panel and Supplementary Figure 6). These data further suggest that LS455 was pyrophosphorylated (two phosphate groups on the same serine residue) by Akt, as seen by the additional enhancement of the 700$\mathrm{nm}$ emission. This result highlights a potentially new role for Akt, which is not known to pyrophosphorylate its substrates. Under similar conditions as LS456 and LS455, Akt did not structurally transform LS631 (Supplementary Figure 5 bottom panel), unless it rapidly formed di-pyrophosphate, which would retain the original spectral profiles of the two emissions.

LS456 reports Akt activation in living cells in real time. Encouraged by the above findings, we then explored the potential of using the DCF contrast technique to image insulin-mediated activation of Akt in living cells. To accomplish this goal, MCF-7 cells were incubated with LS542, LS456, LS455, and LS631 in the presence of $150 \mathrm{nM}$ insulin. The cells were imaged using NIR fluorescence confocal microscopy. The 800-nm fluorescence persisted in LS542 treated cells throughout the experimental period (Figure 2d), but decreased significantly between $0.5 \mathrm{~h}$ and $1 \mathrm{~h}$. Subsequent removal and replacement of insulin with insulin-free culture medium reestablished the red fluorescence intensity to the initial level.

Whereas the $800-\mathrm{nm}$ fluorescence persisted in cells treated with LS542, rapid transformation from 800-nm to 700-nm fluorescence was observed in the cells incubated with LS456 (Figure 2c). Subsequent removal of insulin from the medium by washing the cells and adding insulin-free culture medium reestablished the red fluorescence, indicating the reversibility of the phosphorylation. The reintroduction of insulin in the LS456 culture medium reinitiated the process of red-to-green transformation of the spectral features of the probe. Up to 4 cycles of this transformation were observed when insulin was removed within $20 \mathrm{~min}$ of incubation, and the cells were allowed to incubate in insulin-free medium for $1 \mathrm{~h}$ during each cycle. The insulin-induced DCF cycles in this study were limited by the number of cells remaining after each wash cycle (many cells were lost with each wash). These results suggest that LS456 was phosphorylated in the process and that Akt remains in the active state as long as insulin is present in the culture medium. Thus, the reversibility and 
a

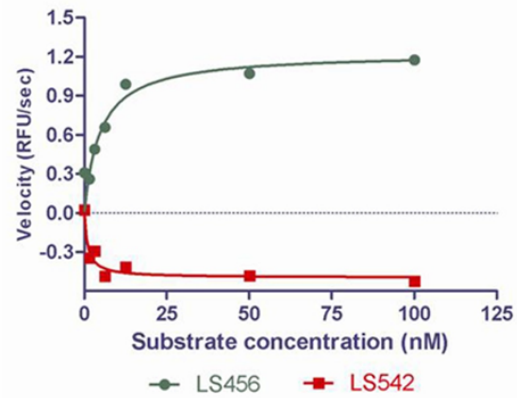

C

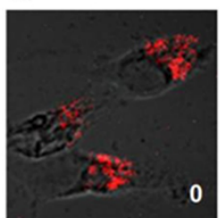

0

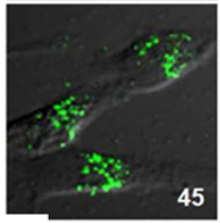

45

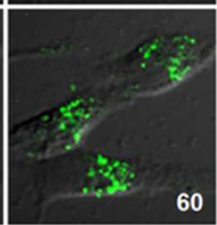

e
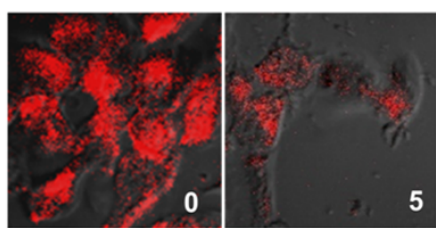

5

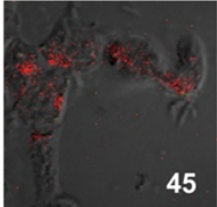

45

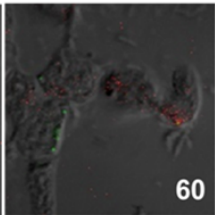

g

$700 \mathrm{~nm}$

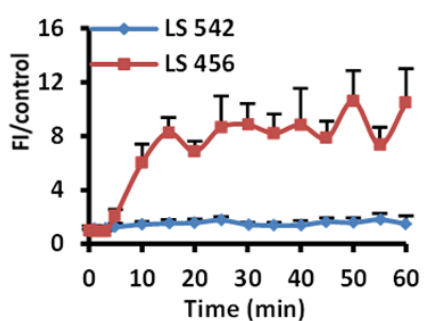

h

b

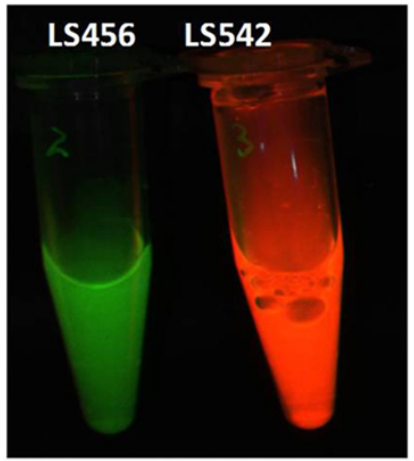

d
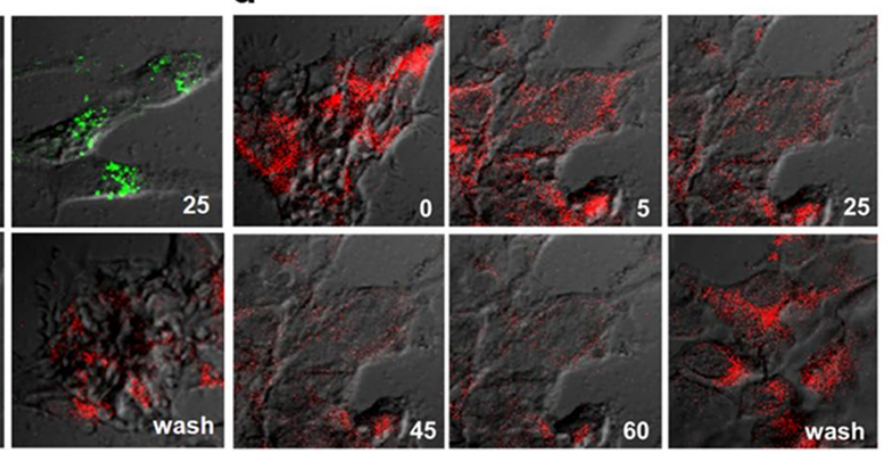

f
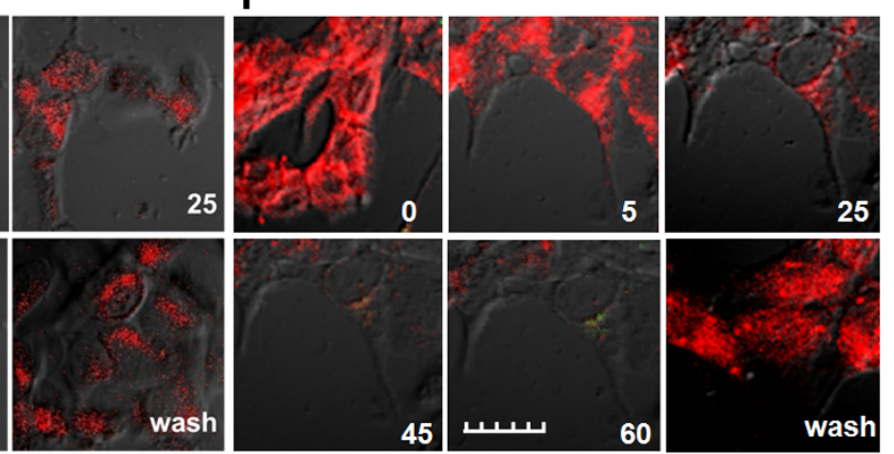

i

$700 \mathrm{~nm} / 800 \mathrm{~nm}$

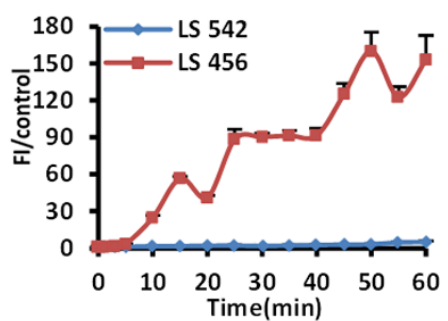

Inhibition (700 nm)

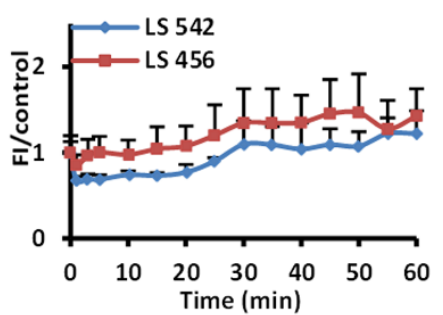

Figure $2 \mid$ Selective phosphorylation of LS456 over LS542 by insulin-activated Akt in MCF-7 cells. (a) In vitro enzyme assay demonstrating the rate of fluorescence increase in the 700-nm channel (green) for LS456, suggesting mono-phosphorylation of the probe. The control compound LS542 had negligible fluorescence changes in both the 700-nm and 800-nm (red, shown) channels. (b) Cell lysate of LS456 and LS542 using monoclonal anti-phosphorylated $\mathrm{Akt}^{473}$ in MCF7 cells after insulin treatment for $20 \mathrm{~min}$. Excitation at 650 and $785 \mathrm{~nm}$ and imaging in both the 700-nm and 800-nm channels show that only LS456 transformed from 800-nm (red) to 700-nm (green) emission, indicating that Akt phosphorylated LS456 but not LS542. (c-f) Real-time NIR fluorescence confocal microscopy of the molecular probe and control compound. The time-dependent transition of LS456 from red to green suggests rapid phosphorylation of the enzyme substrate. The replacement of the insulin solution with non-insulin culture medium demonstrates the reversibility of the process (c). This spectral transition was successfully inhibited with the PI3K inhibitor, wortmannin, in the presence of insulin (d). Similar experiments with LS542 with insulin stimulation (e) and in the presence of wortmannin (f) did not generate any increase in the 700-nm channel. All microscopy studies were conducted at $30^{\circ} \mathrm{C}$. (g-i) Quantitative analysis of the images of panel (c-f). The fluorescence of LS456 at the 700 emission channel increased 10-fold at $50 \mathrm{~min}$ after insulin administration (g). The 700/800-nm fluorescence enhancement ratio of LS456 by insulin stimulation of Akt was $>150$-fold at 50 min post-treatment (h), which was blocked with the PI3K inhibitor wortmannin (i). Red colors represents Ex/Em $=$ $785 \mathrm{~nm} / 805-830 \mathrm{~nm}$. Green colors represents Ex/Em $=633 \mathrm{~nm} / 670-730 \mathrm{~nm}$. Fluorescence images in cells are superimposed on differential interference contrast images. Error bars are reported as mean \pm standard deviation $(\mathrm{N}=8)$. 

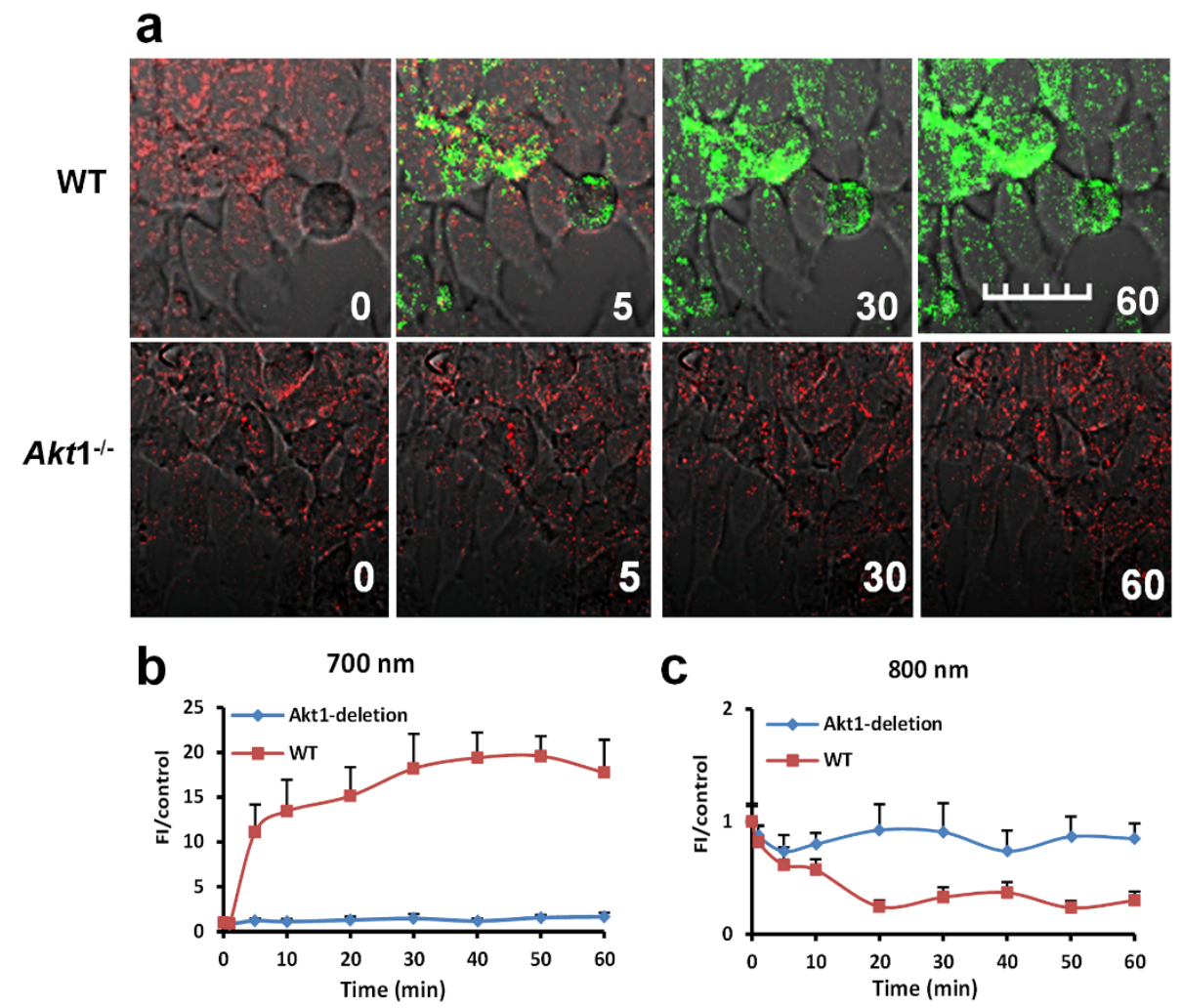

C

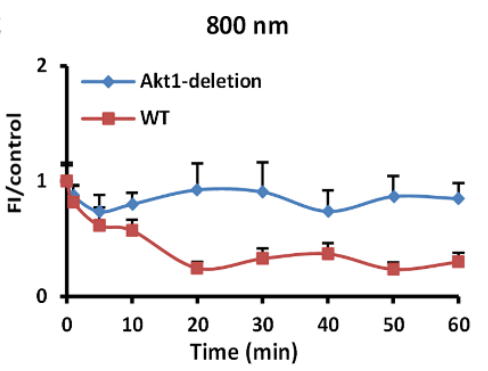

Figure 3 Insulin does not activate the fluorescence enhancement for LS456 at $700 \mathrm{~nm}$ in Akt1-deficient mammary tumor cells. (a) The fluorescence intensity (FI) of LS456 in mammary epithelial cells decreased in the 800-nm channel and increased in the $700 \mathrm{~nm}$ channel, which did not occur in Akt1-deficient cells. (b, c) Quantitative analysis of the FI in WT and Akt1-deficient cells. Fluorescence images in cells are superimposed on differential interference contrast images All figures are at the same scale bars: $20 \mu \mathrm{m}$; error bars are reported as mean \pm standard deviation $(\mathrm{N}=8)$.

longitudinal activation of Akt by new drug inhibitors can be studied by the DCF contrast technique.

Direct quantitative analysis of the DCF intensity changes over time was achieved by acquiring and displaying all of the confocal images on the same intensity scale (Figure $2 \mathrm{~g}-\mathbf{i}$ ). In agreement with the DCF contrast mechanism, there was no fluorescence increase and minimal fluorescence intensity change for the control LS542 in the 700-nm channel (Figure 2g).

In the 800-nm emission channel, the fluorescence signal for both LS456 and LS542 decreased gradually over time, but the rate of signal decrease in cells treated with LS456 was much faster than that of LS542 (Supplementary Figure 7). Thirty minutes after the addition of insulin, the fluorescence intensity of LS456 in the 800nm emission channel decreased 10-fold, whereas that of LS542 decreased 2.5-fold. In all cases, only phosphorylation of LS456 induced a significant fluorescence increase in cells in the 700-nm channel.

LS456 reports the inhibition of Akt phosphorylation potential in cells. To verify that the spectral change was induced by kinasemediated phosphorylation of LS456, we first used a universal kinase inhibitor, K252a, to inhibit LS456 transformation to LS455, as manifested by a $800-\mathrm{nm}$ to $700-\mathrm{nm}$ emission shift. We found that the phosphorylation of LS456 was completely inhibited (not shown). In contrast, the inhibitor did not have a measurable effect on the fluorescence of the control LS542 probe. To further assess the downstream inhibition of Akt, we used wortmannin, a direct inhibitor of PI3K, which is an upstream activator of $\mathrm{Akt}^{17}$. In our in vitro screening assay, we showed that PI3K does not phosphorylate LS456 (Supplementary Figure 3c, Supplementary Figure 3f). Treatment of the MCF-7 cells with wortmannin and either LS456 or LS542, followed by co-incubation with insulin as described above, did not produce significant fluorescence changes in either the 700-nm or 800-nm emission channel (Figure 2e, 2f, 2i). This demonstrates that wortmannin inhibits insulin activation of Akt in MCF-7 cells. Direct evidence of the specificity of Akt phosphorylation of LS456 was obtained by treating MCF-7 cells with an Akt-specific inhibitor, perifosine. This treatment resulted in the inhibition of LS456 phosphorylation, as evidenced by the lack of the 700-nm fluorescence increase (Supplementary Figure 8a).

LS456 specifically reports the activity of the Akt1 isoform in cells. MCF-7 cells express all three isoforms of Akt with distinct subcellular locations ${ }^{18}$. Previous studies have shown that Akt1, Akt2, and Akt3 are localized in the cytosol, mitochondria, and nuclei of breast cancer cells, respectively. On the basis of the predominant cytoplasmic distribution of LS456 in MCF-7 cells, it is logical to anticipate that Akt1 is actively involved in the phosphorylation of LS456. We therefore employed carefully characterized primary mammary epithelial tumor cells derived from MMTV-ErbB2 transgenic mice in either the wild type $\left(A k t^{+/+}\right)$or $A k t 1$ knockout $\left(A k t 1^{-/}\right)$ background $^{19}$ to determine the role of Akt1 in LS456 phosphorylation. The results show that insulin stimulated Akt activity in the $A k t 1^{+/+}$cells, as demonstrated by the transformation of the red to green emission of LS456 (Figure 3). In contrast, LS456 did not respond to insulin treatment in the $A k t 1^{-/}$cells, which also express the Akt 2 and Akt 3 isoforms of the enzyme. Therefore, the large DCF change observed in different cells can be attributed to Akt1. Considering the important role Akt1 plays in pathophysiology and the overarching need to develop small molecule reporters of this isoform, the discovery of LS456 as an Akt1 substrate and selective reporter of its functional status will facilitate the real-time assessment of Akt1 activation in living cells, with the potential to delineate its functions from those of other isoforms in vivo. 


\section{Discussion}

Currently, inefficient, discrete, and low signal-to-noise imaging methods are used to monitor the change in Akt activity in response to insulin stimulation ${ }^{8,9,20}$. Consequently, a new approach, such as the DCF contrast technique, could provide a viable alternative method. The dramatic induction of the DCF change of LS456 by Akt suggests that the compound is a substrate for this enzyme. The consensus substrate motif for Akt has the general peptide sequence Arg-Arg-X$\mathrm{X}-\mathrm{Ser} / \mathrm{Thr}-\varphi$ ( $\varphi$ represents a hydrophobic amino acid), which binds to the catalytic domain of the enzyme through hydrophobic and/or ionic interactions ${ }^{21}$. Although the new substrate lacks the two arginine residues that generally determine the specificity of Akt and the phospho-Ser/Thr binding domain ${ }^{22}$, other studies have shown that the binding between phosphorylated Akt and its substrate could significantly depend on hydrophobic amino acid residues ${ }^{23}$, which is consistent with the general structure of LS456. Particularly, the presence of an elongated and a flexible heptamethine hydrophobic chromophore core of cypate, a positively charged intrinsic iminium ion, and serine residues for phosphorylation most likely allowed LS456 to serve as both a substrate and a reporter for Akt activity.

The exceptionally high detection sensitivity of the 700-nm emission is supported by the spectral properties of the different products, including the relative brightness of the two species contributing to the DCF (Table 1). The relative brightness of LS456 at $700 \mathrm{~nm}$ changed from $20 \%$ to $93 \%$ upon phosphorylation (LS455), accompanied by a significant decrease from $100 \%$ to $33 \%$ in the relative brightness of the $800-\mathrm{nm}$ emission of LS456. Thus, the large change in the spectral properties of the molecular probes demonstrates that DCF is a highly sensitive method for cellular imaging of Akt activity in living cells.

Although interpretation of the DCF for Akt is straightforward, changes in the 700/800 $\mathrm{nm}$ emission ratios were different for other proteins, thereby providing an insight into a potential approach to analyze DCF data. The regeneration of the red fluorescence in cells after a significant decrease following the removal of insulin and cell washing suggests that the fluorescence change in the 800 -nm channel can be attributed to specific interactions between the molecular probe and the kinase. This interpretation only stands when the accompanying changes in the 700-nm emission is incorporated in the analysis. For example, the $700 / 800 \mathrm{~nm}$ vs. time fluorescence ratio is approximately 160 after $50 \mathrm{~min}$ for LS456, compared to the ratiometric change for LS542, which is only approximately 3 (Figure $2 \mathrm{~h}$ ). This sharp ratio increase relative to the moderate change anticipated from the solution spectra of LS456 and LS455 (Figure 1b) is due to the reduction in the $800-\mathrm{nm}$ emission in cells, most likely caused by the binding of these compounds to Akt.

We also postulate that a biological phenomenon that simultaneously increases or decreases the 700/800-nm fluorescence does not reflect a structural transformation of the probe and should not be considered a DCF response. For structures similar to LS456, we can further postulate that mono-, di-, or tri-phosphorylation of the same serine residue is expected to enhance the 700-nm emission, but monophosphorylation of each serine residue to generate a symmetrical diphosphoserine should reverse this effect in a time-dependent DCF change. Furthermore, a decrease in 800 -nm fluorescence with corresponding stable 700-nm fluorescence intensity could be attributed to LS456 interactions with biomolecules. Thus, monitoring the changes in the $800-\mathrm{nm}$ emission could be useful for reporting the extent of molecular interactions in cells. Irrespective of the causative factors that perturb the 800 -nm readout, the Akt-mediated phosphorylation of LS456 results in a significant fluorescence emission shift from $800-\mathrm{nm}$ to $700-\mathrm{nm}$ emissions.

Although a variety of methods is available for ratiometric analysis of data emanating from spectrally distinct emissions, we opted to use a simple ratio of the $700 / 800 \mathrm{~nm}$ relative fluorescence intensity. This approach is particularly suitable for our study because an increase in the $700 \mathrm{~nm}$ emission over a correspondingly small decrease in the $800 \mathrm{~nm}$ channel significantly enhances the contrast. A potential problem with this unconstrained method is the possibility of obtaining disproportionately large ratios if the $800 \mathrm{~nm}$ intensity approaches zero. In this case, an alternative method is to ratio the difference in intensity of the two emissions, followed by dividing the sum of the emissions to constrain the ratiometric change between 1 and -1 . This calculation translates to a ratiometric change of 0.936 and 0.988 at $10 \mathrm{~min}$ and $50 \mathrm{~min}$, respectively, for LS456 (Figure 2 and Supplementary Figure 7). These changes correspond to 30- and 160 - fold increase in the $700 / 800 \mathrm{~nm}$ fluorescence ratio using the simple fluorescence intensity ratiometric approach.

We were intrigued by the possibility that Akt could multiply phosphorylate a single serine residue of LS456, as illustrated in Figure 4. For example, pyrophosphorylation was preferred over the phosphorylation of two individual serine residues (Supplementary Figure 5 and Supplementary Figure 6). Based on the DCF concept, diphosphorylation to give LS631 would revert the 700/800-nm emission of LS455 in cells. Time-lapse cell microscopy with the diphosphoserine LS631 in MCF-7 cells has a similar imaging profile as LS542, suggesting that this compound was not perturbed in vivo or underwent spontaneous pyrophosphorylation on each monophosphoserine (Supplementary Figure 8c). In contrast, LS455 showed an initial co-existence of both red and green fluorescence within 2 min postincubation (Supplementary Figure 8b). After 2 mins, the 700-nm fluorescence increased significantly, reaching a plateau at approximately $20 \mathrm{~min}$. The higher 700 -nm fluorescence remained intact throughout the duration of the study. This trend was quantitatively confirmed as specific to Akt relative to other kinases (Supplementary Figure 5). The 700-nm fluorescence saturation for LS455 (20 min) was much faster than that of LS456 (at $40 \mathrm{~min}$ ) under similar conditions. Unlike LS456, which was dephosphorylated within $60 \mathrm{~min}$ after removing insulin from the culture medium, the dephosphorylation of LS455 was not complete until $270 \mathrm{~min}$ post-removal of insulin.

Recent studies support the potential of Akt to multiply phosphorylate the same serine residue $\mathrm{e}^{19,24,25}$. These studies showed that the binding of ATP to Akt induced an intramolecular interaction between the phosphorylation sites and other domains in the protein, creating a cage around the phosphate group and restricting access of phosphatases to these sites. This interaction favors the pyrophosphorylation and triphosphorylation of small molecules, such as LS456, from the initial monophosphoserine site ${ }^{25}$. Removal of insulin could lead to subsequent uncaging of the binding pocket, which permits the dephosphorylation and inactivation of the kinase.

Another report demonstrated the posttranslational phosphorylation of the monophosphoserine by inositol pyrophosphate kinases ${ }^{26}$. However, we do not believe that these kinases mediated LS456 pyroand tri-phosphorylation because these multiple phosphorylations were also observed in vitro with only Akt. Thus, the DCF probes may provide a new contrast mechanism to distinguish diphosphorylation on two different serine residues from posttranslational pyroor tri-phosphoserine products. These results appear to uncover the potential involvement of Akt in the multi-phosphorylation of its substrates. The next logical step is to assess the native Akt substrates for the presence of pyro- or tri-phosphates.

In this study, the real-time DCF method revealed the reversibility and sustainability of insulin stimulation of Akt. Thus, the dynamic profile of the DCF change with the different compounds studied can be used to uncover intricate molecular events that would otherwise be difficult to observe by current methods. It is also possible to use this approach to discover other substrates for kinases by preparing different Ser/Thr peptides on the dye structural framework that enables the reporting of the functional status of diverse enzymes. To optimize the molecular design, it would be valuable to explore 


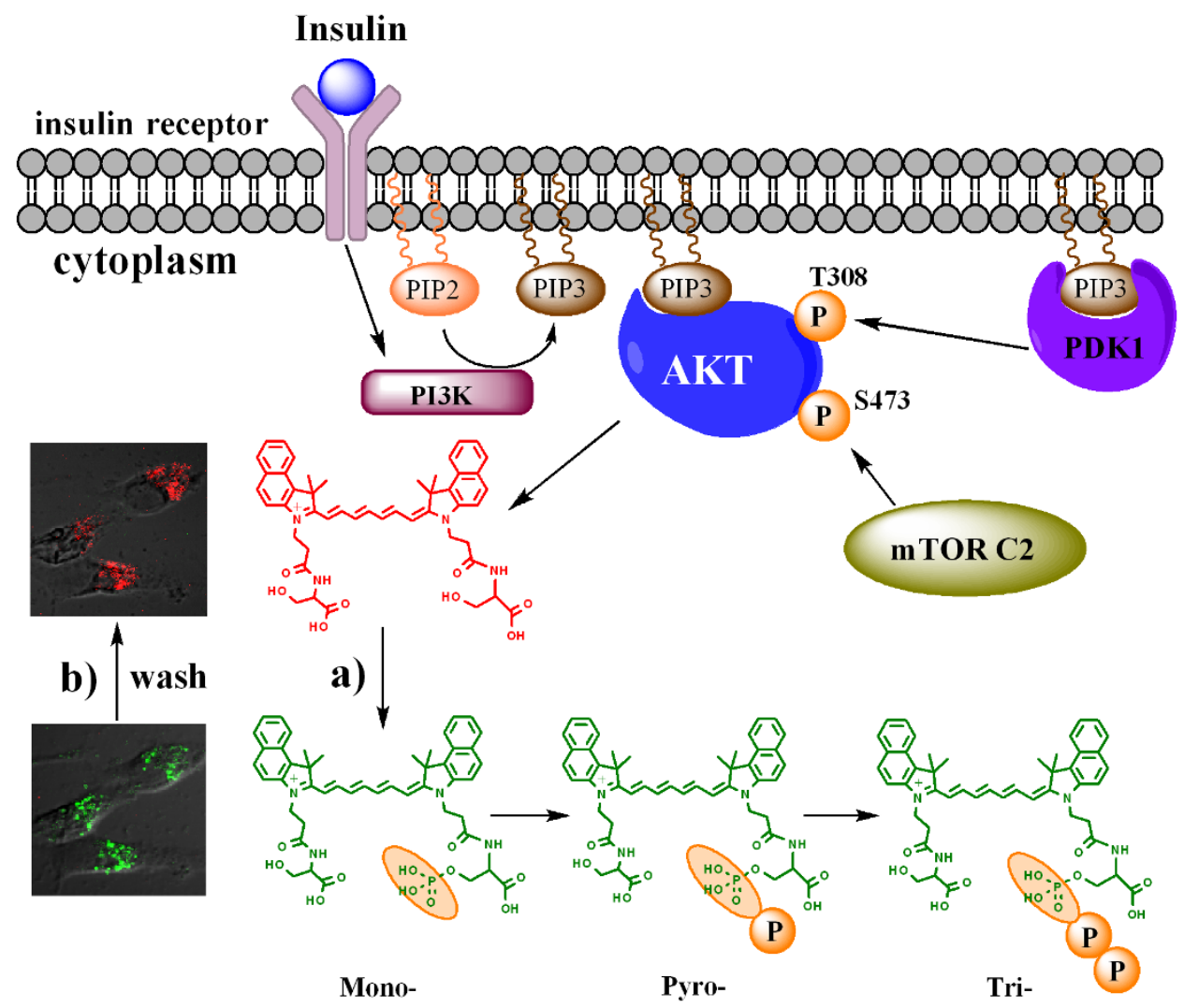

Figure 4 | Illustration of the multiple phosphorylation of LS456 by Akt. The data suggest addition of multiple phosphate groups to one residue of the diserine molecular probe, LS456. After insulin binding to its cell membrane receptors, PI3K was activated to phosphorylate PIP2 into PIP3, which then binds Akt. mTOR complex 2 phosphorylates Akt on the hydrophobic motif ${ }^{473}$ Ser, and the activated Akt phosphorylates LS456 stepwise to form the mono-, pyro-, and tri-phosphoserine sequentially. (a) Insulin-mediated phosphorylation of LS456. (b) De-phosphorylation of mono- and multiplyphosphorylated LS456 after insulin removal. Phosphatidylinositol 3-kinase (PI3K), phosphatidylinositol-3,4,5-trisphosphate (PIP3), mTOR complex 2 (mTOR C2).

the limits of the DCF mechanism by replacing serine with peptide substrates for different kinases.

Although we did not investigate the subcellular localization of the probes, the cytoplasmic distribution of LS456 in MCF-7 cells showed punctate fluorescence (Figure 2c) compared to LS542, which exhibited a more diffuse fluorescence (Figure 2e). The images suggest that the hydrophobic LS542 is primarily localized in the cytosol, while the more hydrophilic LS456 and its phosphorylated derivatives appear to be associated with the outer membranes of intracellular organelles. Therefore, the unique intracellular distribution of different Akt in three distinct intracellular compartments may be responsible for the selective phosphorylation LS456 by Akt1. Unlike the cytosolic Akt1 which has access to LS456 in the cytoplasm, the other two isoforms Akt 2 and Akt 3 are localized in the mitochondria and the nuclei of breast cancer cells, respectively. Because LS456 does not appear to internalize in these organelles, it is likely that the observed selective phosphorylation is mediated by intracellular physical boundaries. Future studies will explore the downstream effect of Akt phosphorylation of LS456 and its potential to serve as an inhibitor of the oncogenic signaling pathway of Akt. To increase the imaging speed, minimize potential photobleaching, and overcome any artifact from fluctuations in the laser power, efforts are in progress to identify the optimal excitation laser for both emissions in cells. Finally, an important advantage of the NIR fluorescence imaging is the low autofluorescence in this spectral region, which improved the detection sensitivity of the DCF method. Coupled with the deep penetration of NIR light in tissue, the NIR fluorescence provides a pathway to translate findings from cells to living organisms, a condition that will ultimately facilitate the use of these probes in humans. This method will be of use in examining patients with cancer, diabetes and other disease because of the association of Akt signaling pathways with these conditions ${ }^{27,28}$.

\section{Methods}

Reagents. O-tert-butyl-L-serine-tert-butyl ester hydrochloride, 2-(1H -benzotriazol1-yl)-1,1,3,3-tetramethyluronium hexafluorophosphate (HBTU), 1hydroxybenzotriazole hydrate (HOBt), $O$-(7-azabenzotriazol-1-yl)- $N, N, N^{\prime}, N^{\prime}$ tetramethyluronium hexafluorophosphate (HATU) and brominated Wang resin were obtained from Nova Biochem (San Diego, CA). $N$ - $\alpha$-Fmoc-O-benzyl-Lphosphoserine was purchased from AnaSpec Inc. (San Jose, CA). Trifluoroacetic acid (TFA), $N, N$-diisopropylcarbodiimide (DIC), $N$-(5-anilino-2,4-pentadienylidene) aniline hydrochloride, 1,1,2-trimethyl-1H-benz[e]indole, 3-bromopropionic acid, cesium iodide (CsI), $N, N$-diisopropylethylamine (DIEA), dichloromethane (DCM), $\mathrm{N}, \mathrm{N}$-dimethylformamide (DMF), ethanol, acetonitrile, hexane, ethyl acetate and dichlorobenzene were purchased from Sigma-Aldrich (St. Louis, MO).

Spectroscopy studies. The absorption and fluorescence spectra of the compounds in methanol were recorded on a Beckman Coulter DU 640 UV-visible spectrophotometer (Beckman Coulter, Inc., Indianapolis, IN) and a Fluorolog-3 spectrofluorometer (Horiba Jobin Yvon, Inc., Edison, NJ), respectively. To capture the dual fluorescence using spectroscopy, the samples were excited at $620 \mathrm{~nm}$ and emission was captured from $635 \mathrm{~nm}$ to $900 \mathrm{~nm}$. The absorption coefficients were calculated from the standard curves at both $780 \mathrm{~nm}$ and $620 \mathrm{~nm}$. The fluorescence quantum yield was measured at $620 \mathrm{~nm}$ excitation, with slit widths set at $5 \mathrm{~nm}$ for both excitation and emission. We calculated the quantum yield by integrating the emission over a range from $650 \mathrm{~nm}$ to $750 \mathrm{~nm}$ for the $700 \mathrm{~nm}$ emission, and from $750 \mathrm{~nm}$ to $850 \mathrm{~nm}$ for the $800 \mathrm{~nm}$ emission at $620 \mathrm{~nm}$ excitation (Table 1). Signal integration was accomplished with FluorEssence software (Horiba Jobin Yvon). Indocyanine Green (ICG) and chlorophyll A were used as reference standards for the $800 \mathrm{~nm}$ and $700 \mathrm{~nm}$ emission, respectively. All measurements were conducted at room temperature. The stability of LS455 and LS456 was evaluated in Tris-saline buffer (TBS, $50 \mathrm{mM}$ Tris; $150 \mathrm{mM} \mathrm{NaCl} ; \mathrm{pH}=7.4$ ) and $150 \mathrm{nM}$ insulin in TBS for $4 \mathrm{~h}$ at $30^{\circ} \mathrm{C}$ using the Fluorolog-3 spectrofluorometer. 
Live cell Akt activity monitoring. MCF-7 cells (ATCC Manassas, VA) were maintained at $37^{\circ} \mathrm{C}$ and $5 \% \mathrm{CO}_{2}$ in DMEM medium supplemented with $10 \%$ fetal bovine serum, $100 \mathrm{unit} / \mathrm{mL}$ penicillin, and $100 \mu \mathrm{g} / \mathrm{mL}$ streptomycin. The cells $(1 \times$ $10^{4}$ cell/well) were cultured in glass bottom dishes (MatTek Co., Ashland, MA 01721) overnight and transfected with $5 \mu \mathrm{M}$ of each LS456, LS455, LS542 using GeneJuice transfection reagent (Novagen, Madison, WI) in the dish for $18 \mathrm{~h}$ at $37^{\circ} \mathrm{C}$, according to the manufacturer's instructions. The transfected cells were treated with $150 \mathrm{nM}$ insulin in Tris-buffered saline and imaged at $30^{\circ} \mathrm{C}$ using an FV1000 confocal microscope with UPLanApo/IR 60X/1.20W objective lens (Olympus, Center Valley, PA). After washing 3 times for $5 \mathrm{~min}$ in TBS, the cells were pretreated for $1 \mathrm{~h}$ with $1 \mu \mathrm{M}$ wortmannin PI3K inhibitor (Cell signaling Technology, Inc., Boston, MA) or for $2 \mathrm{~h}$ with $50 \mu \mathrm{M}$ Akt inhibitor perifosine (Selleckchem, Inc., Houston, TX). The treated cells were incubated with $150 \mathrm{nM}$ of insulin and $10 \mu \mathrm{M}$ wortmannin or $50 \mu \mathrm{M}$ perifosine in TBS, respectively. Imaging was conducted at different time points. The mean fluorescence intensity $(n=8)(E x / E m=633 / 670-730 \mathrm{~nm} ; \mathrm{Ex} / \mathrm{Em}$ $=785 / 805-830 \mathrm{~nm}$ ) in the dishes was determined with FV1000 software. All of the fluorescence intensity changes at each time point were normalized to the positive control ( $0 \mathrm{~min}$ ). The image of the tubes containing LS456 and LS542 (Figure 2b) were acquired with LI-COR Pearl Imager (Lincoln, NE) at Ex/Em of 685/705 nm and $785 / 810 \mathrm{~nm}$ channels. Live cell imaging studies were conducted at $30^{\circ} \mathrm{C}$, which is the default room temperature for the imaging platform of our microscope. All images within the same time series were recorded from the same area of the slides. However, the live cells moved during the imaging sessions, resulting in variability in the total number of cells displayed in Figure 2c-f. To account for these changes in the field of view, eight representative cells were used to quantify the fluorescence using Olympus FV1000 software. The data shown in Figure $2 \mathrm{~g}-\mathbf{i}$ were obtained from a duplicate of 4different experiments.

Comparison of Akt activity from WT and Akt1-deficient cells. The Akt1 $1^{-1-}$ and wild type (WT) cells used in this study were cultured and transfected as previously described ${ }^{19}$. Briefly, mammary tumor epithelial cells derived from MMTV-ErbB2 transgenic mice tumors were cultured in F-12 medium (Sigma, St. Louis, MO) with EGF $(10 \mathrm{ng} / \mathrm{mL})$, hydrocortisone $(1 \mu \mathrm{g} / \mathrm{mL})$, penicillin (100 units $/ \mathrm{mL})$, streptomycin $(100 \mu \mathrm{g} / \mathrm{mL})$, and gentamycin $(50 \mu \mathrm{g} / \mathrm{mL})$ and supplemented with $10 \%$ FBS. EGF was obtained from Sigma. The $A k t 1^{-1-}$ and WT cells $\left(1 \times 10^{3}\right.$ cell/well $)$ were cultured in glass bottom dishes and were transfected and imaged in real-time at $30^{\circ} \mathrm{C}$ using confocal microscopy at different time points. The mean fluorescence intensity of the cells $(n=8)(E x / E m=633 / 670-730 n m ; E x / E m=633 / 731-$ $800 \mathrm{~nm}$ ) in the dishes was determined with the FV1000 software and normalized to FI at the initiation step $(0 \mathrm{~min})$.

In vitro phosphorylation assay. Our probes and the Sox reference peptide (Invitrogen, Carlsbad, CA) were dissolved in assay buffer (20 mM HEPES, pH 7.0, $10 \mathrm{mM} \mathrm{CaCl}_{2}, 0.05 \%$ Brij-35). Different concentrations of probes were mixed with $100 \mathrm{ng}$ of different enzymes and $1 \mathrm{mM}$ ATP (Sigma, St. Louis, MO) in $50 \mu \mathrm{L}$ assay buffer at $30^{\circ} \mathrm{C}$. The Sox peptide was also mixed with $100 \mathrm{ng}$ of Akt according to the manufacturer's instructions. Fluorescence intensity was measured in real time on a microplate reader (Synergy HT, BioTek Instruments, Inc., Winooski, VT) at $\lambda_{\text {excitation }}$ $=620 \pm 20 \mathrm{~nm} ; \lambda_{\text {emission }}=700 \pm 10 \mathrm{~nm}$ and $\lambda_{\text {excitation }}=760 \pm 30 \mathrm{~nm} ; \lambda_{\text {emission }}=$ $810 \pm 20 \mathrm{~nm}$. Initial velocities were determined by fitting the initial linear section of the curve obtained from plots of fluorescence intensity versus time. These initial velocities were plotted with respect to the substrate concentration.

1. Noble, M. E., Endicott, J. A. \& Johnson, L. N. Protein kinase inhibitors: insights into drug design from structure. Science 303, 1800-5 (2004).

2. Lawlor, M. A. \& Alessi, D. R. PKB/Akt: a key mediator of cell proliferation, survival and insulin responses? J Cell Sci 114, 2903-10 (2001).

3. Yaffe, M. B. et al. A motif-based profile scanning approach for genome-wide prediction of signaling pathways. Nat Biotechnol 19, 348-53 (2001).

4. Hers, I., Vincent, E. E. \& Tavare, J. M. Akt signalling in health and disease. Cell Signal 23, 1515-27.

5. Chin, Y. R. \& Toker, A. Akt isoform-specific signaling in breast cancer: uncovering an anti-migratory role for palladin. Cell Adh Migr 5, 211-4.

6. Santi, S. A. \& Lee, H. The Akt isoforms are present at distinct subcellular locations. Am J Physiol Cell Physiol 298, C580-91.

7. Sekar, R. B. \& Periasamy, A. Fluorescence resonance energy transfer (FRET) microscopy imaging of live cell protein localizations. J Cell Biol 160, 629-33 (2003).

8. Sasaki, K., Sato, M. \& Umezawa, Y. Fluorescent indicators for Akt/protein kinase $\mathrm{B}$ and dynamics of Akt activity visualized in living cells. J Biol Chem 278, 3094551 (2003).

9. Kenworthy, A. K. Imaging protein-protein interactions using fluorescence resonance energy transfer microscopy. Methods 24, 289-96 (2001).

10. Zhang, Z. et al. Near-infrared dichromic fluorescent carbocyanine molecules. Angew Chem Int Ed Engl 47, 3584-7 (2008).
11. Achilefu, S., Dorshow, R. B., Bugaj, J. E. \& Rajagopalan, R. Novel receptor-targeted fluorescent contrast agents for in vivo tumor imaging. Invest Radiol 35, 479-85 (2000).

12. Lim, K. H., Ancrile, B. B., Kashatus, D. F. \& Counter, C. M. Tumour maintenance is mediated by eNOS. Nature 452, 646-9 (2008).

13. Tokunaga, E. et al. Deregulation of the Akt pathway in human cancer. Curr Cancer Drug Targets 8, 27-36 (2008).

14. Karlsson, H. K. et al. Insulin-stimulated phosphorylation of the Akt substrate AS160 is impaired in skeletal muscle of type 2 diabetic subjects. Diabetes 54, 1692 7 (2005).

15. Song, G., Ouyang, G. \& Bao, S. The activation of Akt/PKB signaling pathway and cell survival. J Cell Mol Med 9, 59-71 (2005).

16. Mayo, L. D. \& Donner, D. B. A phosphatidylinositol 3-kinase/Akt pathway promotes translocation of $\mathrm{Mdm} 2$ from the cytoplasm to the nucleus. Proc Natl Acad Sci U S A 98, 11598-603 (2001).

17. Coffer, P. J., Jin, J. \& Woodgett, J. R. Protein kinase B (c-Akt): a multifunctional mediator of phosphatidylinositol 3-kinase activation. Biochem J 335 (Pt 1), 1-13 (1998).

18. Santi, S. A. \& Lee, H. The Akt isoforms are present at distinct subcellular locations. Am J Physiol Cell Physiol 298, C580-91 (2010).

19. Ju, X. et al. Akt1 governs breast cancer progression in vivo. Proc Natl Acad Sci U S A 104, 7438-43 (2007).

20. Kunkel, M. T., Ni, Q., Tsien, R. Y., Zhang, J. \& Newton, A. C. Spatio-temporal dynamics of protein kinase $\mathrm{B} /$ Akt signaling revealed by a genetically encoded fluorescent reporter. J Biol Chem 280, 5581-7 (2005).

21. Calleja, V., Laguerre, M. \& Larijani, B. 3-D structure and dynamics of protein kinase B-new mechanism for the allosteric regulation of an AGC kinase. J Chem Biol 2, 11-25 (2009).

22. Obata, T. et al. Peptide and protein library screening defines optimal substrate motifs for AKT/PKB. J Biol Chem 275, 36108-15 (2000).

23. Shults, M. D., Carrico-Moniz, D. \& Imperiali, B. Optimal Sox-based fluorescent chemosensor design for serine/threonine protein kinases. Anal Biochem 352, 198-207 (2006).

24. Lin, K. et al. An ATP-site on-off switch that restricts phosphatase accessibility of Akt. Sci Signal 5, ra37.

25. Humphrey, S. J. \& James, D. E. Uncaging akt. Sci Signal 5, pe20.

26. Bhandari, R. et al. Protein pyrophosphorylation by inositol pyrophosphates is a posttranslational event. Proc Natl Acad Sci U S A 104, 15305-10 (2007).

27. Zoncu, R., Efeyan, A. \& Sabatini, D. M. mTOR: from growth signal integration to cancer, diabetes and ageing. Nat Rev Mol Cell Biol 12, 21-35 (2011).

28. Vasudevan, K. M. \& Garraway, L. A. AKT signaling in physiology and disease. Curr Top Microbiol Immunol 347, 105-33 (2010).

\section{Acknowledgements}

We thank Mikhail Berezin and Sharon Bloch (Radiology) for useful discussions, as well as Fongfu Hsu and Alan N. Bohrer (Internal Medicine) for technical assistance with MS/MS analysis. This work was supported in part by grants from the National Institutes of Health (NIBIB R01 EB008111 and R01 EB008458; and NCI R01 CA171651) and the National Science Foundation (CCF 0963742). The content is solely the responsibility of the authors and does not necessarily represent the official views of the National Institutes of Health.

\section{Author contributions}

M.B. and R.T. prepared the compounds and determined spectral properties; D.S. conducted confocal microscopy, Co-IP and gel extraction experiments; B.X. performed the enzyme assay; S.A. and D.S. designed the experiments; X.J. and R.G.P. provided Akt1 $1^{-1 /}$ and W.T. cells; S.A. designed compounds and conceived biological applications; D.S. and S.A. conceived application to Akt and designed biological experiments; D.S., M.B., R.T., B.X. and S.A. analyzed data; D.S., M.B., R.T., B.X., R.G.P. and S.A. participated in writing the manuscript

\section{Additional information}

Supplementary information accompanies this paper at http://www.nature.com/ scientificreports

Competing financial interests: The authors declare no competing financial interests.

License: This work is licensed under a Creative Commons

Attribution-NonCommercial-NoDerivs 3.0 Unported License. To view a copy of this license, visit http://creativecommons.org/licenses/by-nc-nd/3.0/

How to cite this article: Shen, D.W. et al. Dual fluorescent molecular substrates selectively report the activation, sustainability and reversibility of cellular PKB/Akt activity. Sci. Rep. 3 , 1697; DOI:10.1038/srep01697 (2013) 